\title{
Intoxicações naturais por plantas em ruminantes diagnosticadas no Setor de Patologia Veterinária da UFRGS no período de 1996-2005
}

\author{
Spontaneous poisoning in ruminants by consumption of toxic plants: \\ cases diagnosed by the Laboratory of Veterinary Pathology (UFRGS) in the period 1996-2005 \\ Pedro Miguel Ocampos Pedroso, Caroline Argenta Pescador, Eduardo Conceição de Oliveira, \\ Luciana Sonne, Paulo Mota Bandarra, Djeison Lutier Raymundo \& David Driemeier
}

\begin{abstract}
RESUMO
As intoxicações por plantas em animais pecuários têm particular importância em áreas onde o manejo do pastoreio é feito de forma extensiva. Entre os herbívoros, os ruminantes são os mais suscetíveis. Esse estudo retrospectivo incluiu as intoxicações por plantas em ruminantes diagnosticadas no Setor de Patologia Veterinária (SPV) da Universidade Federal do Rio Grande do Sul (UFRGS) no período de 1996-2005. Essas intoxicações causaram 7,58\% (43/567) das mortes de bovinos, $11,46 \%$ (25/218) de ovinos e 7,69 \% (16/208) de caprinos necropsiados pelo SPV-UFRGS. Destaca-se que das perdas associadas com intoxicações: 62,79\% das mortes em bovinos foram atribuídas à lesão hepática crônica causada por Senecio spp.; $28 \%$ das mortes em ovinos, às lesões no trato gastrintestinal por Baccharis coridifolia e 75\% das mortes em caprinos, à doença de depósito lisossomal por Sida carpinifolia. Os achados indicam que a intoxicação por plantas é importante causa de perda econômica em ruminantes na área de atuação do SPV/UFRGS no Rio Grande do Sul.
\end{abstract}

Descritores: plantas tóxicas, ruminantes, bovino, ovino, caprino.

\section{ABSTRACT}

Spontaneous poisoning by toxic plants in farm animals has particular importance in areas where extensive pastoral management is practiced. Among herbivores, ruminants are the most susceptible species. This retrospective study includes the cases of plant poisoning in ruminants diagnosed in the Laboratory of Veterinary Pathology, Federal University of Rio Grande do Sul (UFRGS) in the period 1996-2005. These conditions caused 7.58\% (43/567) of the deaths in cattle; 11.46\% (25/218), in sheep, and 7.69\% (16/208) in goats. Among deaths caused by plant poisoning, Senecio spp., which causes chronic hepatic lesions, was responsible for $62.79 \%$ of them in cattle; Baccharis coridifolia, which causes lesions in the gastrontestinal tract, was linked to $28 \%$ of the deaths in sheep, and Sida carpinifolia that causes lysosomal storage disease was associated with $75 \%$ of deaths in goats. These data indicate that poisonous plants are important causes of economic losses in ruminant in Rio Grande do Sul.

Keywords: plant poisoning, ruminants, cattle, sheep, goat. 


\section{INTRODUÇÃO}

As intoxicações por plantas tóxicas em animais pecuários têm particular importância em áreas em que se pratica pastoreio extensivo [12]. Define-se como planta tóxica de interesse pecuário aquelas que quando ingeridas pelos animais domésticos, sob condições naturais, causam danos à saúde ou mesmo a morte [14,25]. No Brasil, estão identificadas cerca de 88 espécies de plantas tóxicas para os animais [20]. Estima-se que $10 \%$ a $14 \%$ das mortes em bovinos e $15 \%$ a $20 \%$ das mortes em ovinos sejam causadas por plantas tóxicas anualmente no Rio Grande do Sul [13]. O consumo dessas plantas por animais domésticos é determinado por diversos fatores que incluem palatabilidade, carência de forragens, longos períodos de privação hídrica e introdução de animais (mantidos em locais onde não ocorre a planta) em pastagens desconhecidas [14]. Nesse estudo retrospectivo, são descritos os achados observados em intoxicações de ruminantes por plantas diagnosticadas no SPV-UFRGS, no período de 1996-2005.

\section{MATERIAIS E MÉTODOS}

Foram revisados os arquivos de necropsias de bovinos, ovinos e caprinos acometidos por intoxicações por plantas tóxicas no período de 1996-2005.
Os dados epidemiológicos, de histórico e sinais clínicos foram obtidos com veterinários e proprietários e acompanhados pela equipe do SPV em visitas às propriedades, ocasião em que também se fez a caracterização de achados macroscópicos. Fragmentos de órgãos foram fixados em formol a 10\%, processados pelos métodos histológicos de rotina e corados por Hematoxilina e eosina (HE) [11], bem como por outras colorações especiais, quando necessário. Adicionalmente, estudos experimentais foram realizados para comprovação de toxicidade de plantas.

\section{RESULTADOS}

No período analisado, foram necropsiados 567 bovinos, 218 ovinos e 208 caprinos. As intoxicações por plantas tóxicas foram responsáveis por $7,58 \%$ (43/567) das mortes em bovinos; 11,46\% (25/218) em ovinos e 7,69\% (16/208) em caprinos. As principais plantas causadoras de mortes em ruminantes foram as hepatotóxicas (especialmente o Senecio spp.), as que afetam o tubo digestivo (Baccharis coridifolia) e as que causam doença de depósito lisossomal (Sida carpinifolia). A Tabela 1 resume as intoxicações por plantas em ruminantes diagnosticadas no SPV no período 1996-2005.

\begin{tabular}{|c|c|c|c|}
\hline Plantas tóxicas & Bovinos (\%) & Ovinos (\%) & Caprinos (\%) \\
\hline Senecio spp. & 62,79 & * & * \\
\hline Baccharis coridifolia & 9,3 & 28 & * \\
\hline Mascagnia spp. & 9,3 & 8 & * \\
\hline Pteridium aquilinum & 4,65 & * & * \\
\hline Xanthium cavanillesii & 4,65 & * & * \\
\hline Trema micrantha & 2,32 & * & 6,25 \\
\hline Sida carpinifolia & 2,32 & 24 & 75 \\
\hline Vicia spp. & 2,32 & * & * \\
\hline Erythroxylum deciduum & * & 24 & * \\
\hline Brachiaria decumbens & * & 8 & * \\
\hline Cynodon sp & * & 8 & 6,25 \\
\hline Prunus sphaerocarpa & * & * & 12,5 \\
\hline
\end{tabular}




\section{Intoxicações em Bovinos}

O total de $62,79 \%$ (27/43) das mortes de bovinos por intoxicações por plantas no período foi atribuído à lesão hepática crônica causada por Senecio spp. A espécie de Senecio spp. mais encontrada foi $S$. brasiliensis. Em menor quantidades, estiveram $S$. oxyphyllus e $S$. selloi. Os sinais clínicos mais freqüentemente associados com a doença foram emagrecimento progressivo, incoordenação, diarréia, tenesmo, prolapso retal e agressividade. Os achados de necropsia mais comuns foram fígado aumentado de tamanho e endurecido, vesícula biliar aumentada e com aspecto grumoso, edema no abomaso e mesentério, assim como acúmulo de líquido nas cavidades abdominal e torácica. Os principais achados microscópicos foram fibrose hepática difusa acentuada (23/ $27-85,18 \%)$, megalocitose (17/27-62,96\%), proliferação de ductos biliares (17/27-62,96\%), necrose individual de hepatócitos (4/27-14,81\%), tumefação hepatocelular e colestase ( 2 casos-7,4\%). Havia também espongiose no sistema nervoso central $(8 / 27$ $29,62 \%)$, congestão pulmonar (3/27-11,11\%), edema pulmonar (1/27-3,7\%), edema de abomaso (2/27-7,4\%), hiperplasia linfóide, edema de intestino e tumefação de túbulos renais, cada um com 1 caso $(3,7 \%)$. Outras plantas que causaram morte em bovinos foram Baccharis coridifolia (4/43-9,3\%), Mascagnia spp. (4/43-9,3\%), Pteridium aquilinum e Xanthium cavanillesii (2/434,65\%) cada um, Sida carpinifolia, Trema micrantha e Vicia spp., cada uma com 1 caso (2,32\%).

\section{Intoxicações em Ovinos}

A intoxicação por $B$. coridifolia, que causa lesões no trato gastrintestinal, representou $28 \%(7 / 25)$ das mortes na espécie. Os achados de necropsia mais frequientes nesses casos foram edema da parede ruminal $(6 / 7-85,71 \%)$, coloração avermelhada na submucosa $(6 / 7-85,71 \%)$ e na mucosa do abomaso (6/ 7-85,71\%). As principais lesões microscópicas observadas incluíram necrose do epitélio da mucosa do rúmen (6/7-85,71\%), baço congesto (2/7-28,57\%), abomaso congesto, hemorrágico e com edema na submucosa (1/7-14,28\%), hepatite multifocal (2/7$28,57 \%$ ), congestão hepática, degeneração gordurosa, tumefação e necrose individual de hepatócitos (1/7$14,28 \%)$, necrose de mucosa intestinal (1/7-14,28\%), nefrite intersticial (1/7-14,28\%) e congestão e hemorragia pulmonares (1/7-14,28\%). Na segunda posição, estiveram simultaneamente Erythroxylum deciduum e $S$. carpinifolia, com 6 casos cada $(6 / 25-24 \%)$. Os principais sinais clínicos observados na intoxicação por S. carpinifolia foram sinais neurológicos, tremores musculares, ataxia e definhamento. Aumento de volume dos linfonodos mesentéricos foi a única alteração nas necropsias. As principais alterações histológicas foram vacuolização citoplasmática, especialmente das células de Purkinje do cerebelo, dos neurônios do córtex cerebral, do mesencéfalo, do tálamo, cornos ventrais da medula espinhal, esferóides axonais no encéfalo e na camada granular do cerebelo. Outras vacuolizações citoplasmáticas foram observadas no epitélio dos túbulos renais, ácinos pancreáticos, células foliculares da tireóide, hepatócitos e macrófagos de órgãos linfóides.

A intoxicação por E. deciduum (cocão) em ovinos (6/25-24\%) foi causada pela ingestão de frutos da planta e caracterizou-se por hemorragia nas serosas, edema pulmonar e presença de grande quantidade de sementes e frutos inteiros da planta no conteúdo ruminal. Não havia alterações microscópicas significativas nos órgãos examinados. Outras intoxicações diagnosticadas em ovinos foram Mascagnia spp., Brachiaria decumbens e Cynodon sp, cada um com $8 \%(2 / 25)$.

\section{Intoxicações em Caprinos}

S. carpinifolia, que causa doença de depósito lisossomal, representou 75\% (12/16) dos diagnósticos de intoxicação por plantas em caprinos. Todos esses 12 animais apresentaram incoordenação, tremores musculares, opistótono, nistagmo e quedas à manipulação. Apenas os linfonodos tinham alterações à necropsia. As lesões histológicas foram caracterizadas por vacuolizações citoplasmáticas nas células de Purkinje do cerebelo, neurônios do mesencéfalo, córtex cerebral, do tálamo e dos cornos ventrais da medula espinhal. A segunda causa de intoxicação por plantas em caprinos foi Prunus sphaerocarpa, representando 12,5\% (2/16). Esses dois casos apresentaram folhas da planta na entrada do cárdia e misturadas com o bolo alimentar no rúmen. Os demais órgãos não apresentaram alterações. Não havia alterações histológicas significativas. Cynodon sp e T. micrantha causaram uma morte cada uma (1/16-6,25\%).

\section{DISCUSSÃO}

Esse estudo apresenta a casuística de intoxicações causadas por plantas em ruminantes da rotina 
laboratorial do SPV-UFRGS no período analisado. Obviamente, diferenças podem ser constatadas em comparações com dados de outros laboratórios de diagnóstico veterinário. Distorções podem ocorrer, em parte, devido à ênfase gerada em determinados projetos de pesquisa desenvolvidos no SPV no período em questão. As perdas não se atribuem somente às mortes, mas também aos gastos com medicamentos e às baixas de produtividade dos animais. Entre as espécies de plantas que ainda não haviam sido associadas com intoxicações estiveram S. carpinifolia, $T$. micrantha e E. deciduum. No Rio Grande do Sul, a intoxicação por Senecio spp. é a segunda causa de morte em bovinos (aproximadamente $7 \%$ de todas as mortes), sendo superada somente pela tristeza parasitária [7]. A planta se distribui por todo o Estado do Rio Grande do Sul e sua ingestão ocorre entre os meses de maio e agosto, período em que há maior carência de forragem e quando as diversas espécies de Senecio spp. estão em brotação [18]. Intoxicação por Senecio spp. em bovinos provoca perdas econômicas importantes pela queda na produtividade dos animais e morte, pois os alcalóides pirrolizidínicos, princípio ativo da planta, determinam uma lesão irreversível e crônica no fígado dos animais que a consomem [6,9,10]. No período 1996-2005 a doença foi mais frequiente em fêmeas $(22-81,48 \%)$ do que machos $(5-18,51 \%)$. Essa observação pode estar associada com a maior permanência das vacas em certos sistemas de criação, uma vez que a doença tem caráter crônico [9], ou apenas com o maior número daquelas em outros sistemas de exploração pecuária. Durante o período analisado não se constatou intoxicação por Senecio spp. em ovinos. Nas propriedades onde ocorreu a intoxicação não havia criação simultânea de ovinos e bovinos. Os sinais da intoxicação são primariamente associados com a lesão hepática e podem aparecer vários meses após o animal ter ingerido a planta pela última vez [1], ou até dois anos após a ingestão [5]. O quadro clínico, assim como os achados de necropsia e histopatológicos observados coincidem com os descritos por vários autores [1,7-10,25]. A ocorrência de diferentes espécies de Senecio spp. nas propriedades, as manifestações clínicas e patológicas observadas pelos veterinários de campo e pela equipe do SPV-UFRGS foram compatíveis com o diagnóstico de intoxicação por alcalóides pirrolizidínicos causada pela ingestão de plantas do gênero Senecio.
Na intoxicação por B. coridifolia em ovinos, o diagnóstico foi fundamentado pelas lesões de necropsia, lesões microscópicas e dados epidemiológicos, sendo que dos 7 animais necropsiados, 6 ocorreram em um único surto e outro foi um caso isolado. Os animais intoxicados são aqueles introduzidos em áreas onde há a planta e são provenientes de regiões onde a planta não ocorre, exatamente como observado nos casos aqui mencionados [17]. Os achados macro e microscópicos mais freqüentes aqui relatados foram os mesmos descritos em bovinos por outros autores [16,25].

Na intoxicação por S. carpinifolia em ovinos, a vacuolização citoplasmática de neurônios e células gliais foram os achados histológicos mais significativos, assim como descrito anteriormente [19]. A vacuolização neuronal é difusa, sendo mais evidente nas células de Purkinge do cerebelo [15]. Os sinais clínicos, achados macroscópicos e microscópicos são similares aos descritos em caprinos [2,4], porém os sinais clínicos são menos evidentes que em caprinos [2].

Intoxicação natural com morte de caprinos por Sida carpinifolia e subsequiente comprovação experimental foi descrita inicialmente por Driemeier et al. [4]. Na intoxicação natural de caprinos por $S$. carpinifolia, todos animais necropsiados eram mantidos em potreiros cuja vegetação predominante era $S$. carpinifolia. Os achados clínicos e patológicos dessa intoxicação foram semelhantes descritos nos casos envolvendo plantas dos gêneros Swainsona, Oxytropis, Astragalus e Ipomoea [2,4,25]. Distensão e vacuolização citoplasmáticas, principalmente nas células de Purkinge do cerebelo, neurônios do córtex cerebral, tálamo, mesencéfalo e medula espinhal foram relatados previamente [2]. A intoxicação por E. deciduum em ovinos também foi observada primeiramente por técnicos do SPV-UFRGS e relatada posteriormente por Colodel et al. [3].

A toxicidade de Trema micrantha foi observada experimentalmente em coelhos [24], bovinos [23] e caprinos [22], espécie em também foi comprovada a intoxicação natural [21]. Lesões macroscópicas em caprinos incluem hemorragias subcutâneas no esterno, fígado com coloração amarelada, aspecto friável, padrão lobular evidente e áreas vermelhas entremeadas com áreas mais claras na superfície de corte. Achados microscópicos caracterizam-se por necrose hepática centrolobular [21,22]. O diagnóstico de intoxicação por T. micrantha nos caprinos baseou-se na presença da planta no local das mortes, ausência de outras plantas 
hepatotóxicas, além das lesões macroscópicas e microscópicas semelhantes às observadas na reprodução experimental na espécie caprina [22].

\section{CONCLUSÕES}

Esse estudo indica que as plantas tóxicas constituem-se em importante causa de perdas por mortes em ruminantes na região de atuação do SPV-UFRGS.
Agradecimentos. Os autores agradecem aos Professores Edson Moleta Colodel, Alexandre Paulino Loretti, Sandra Davi Traverso e Cláudio Estêvão Farias da Cruz pelo auxílio no diagnóstico e nas descrições do material de arquivo pesquisado.

\section{REFERÊNCIAS}

1 Barros C.S.L., Metzdorf L.L. \& Peixoto P.V. 1987. Ocorrência de surtos de intoxicação por Senecio spp. (Compositae) em bovinos no Rio Grande do Sul. Pesquisa Veterinária Brasileira. 7: 101-107.

2 Colodel E.M., Driemeier D., Loretti A.P.,Gimeno E.J., Traverso S.D., Seitz A.L. \& Zlotowski P. 2002. Aspectos clínicos e patológicos da intoxicação por Sida carpinifolia (Malvaceae) em caprinos no Rio Grande do Sul. Pesquisa Veterinária Brasileira. 22: 51-57.

3 Colodel E.M., Seitz A.L., Schmitz M., Borba M.R., Raymundo D.L. \& Driemeier D. 2004. Intoxicação por Erythroxylum deciduum (Erythroxylaceae) em ovinos. Pesquisa Veterinária Brasileira. 24: 165-168.

4 Driemeier D., Colodel E.M., Gimeno E.J. \& Barros S.S. 2000. Lysosomal storage disease caused by Sida carpinifolia poisoning in goats. Veterinary Pathology. 37: 153-159.

5 Gava A., Pilati C., Mendez R.E. \& Neves D.S. 2004. Intoxicação por Senecio brasiliensis em bovinos, no Estado de Santa Catarina: formas de ingestão, sinais clínicos e lesões. Pesquisa Veterinária Brasileira. 24 (Supl): 26-27.

6 Karam F.S.C., Méndez M.C., Jarenkow J.A. \& Riet-Correa F. 2002. Fenologia de quatro espécies tóxicas de Senecio (Asteraceae) na região Sul do Rio Grande do Sul. Pesquisa Veterinária Brasileira. 22: 33-39.

7 Karam F.S.C., Soares M.P., Haraguchi M., Riet-Correa F., Méndez M.C. \& Jarenkow J.A. 2004. Aspectos epidemiológicos da seneciose na região sul do Rio Grande do Sul. Pesquisa Veterinária Brasileira. 24: 191-198.

8 Kelly W.R. 1993. The liver and biliary system. In: Jubb K.V.F., Kennedy P.C. \& Palmer N. (Eds). Pathology of Domestic Animals. v.2. 4th edn. London: Academic Press, pp.319-406.

9 Méndez M.C. \& Riet-Correa F. 2000. Plantas hepatotóxicas. In: Méndez M.C. \& Riet-Correa F. (Eds). Plantas tóxicas e micotoxicoses. Pelotas: Editora Universitária, pp.9-29.

10 Méndez M.C., Riet-Correa F. \& Schild A.L. 1987. Intoxicação por Senecio spp. (Compositae) em bovinos no Rio Grande do Sul. Pesquisa Veterinária Brasileira. 7: 51-56.

11 Prophet E.B., Mills B., Arrington J.B. \& Sobin L.H. 1992. Laboratory Methods in Histotechnology. Washington: American Registry of Pathology, 279p.

12 Radostits O.M., Gay. C.C., Blood D.C. \& Hinchcliff K.W. 2002. Doenças causadas por toxinas de plantas, fungos, cianofitas, clavibactéria e por venenos de carrapatos e animais vertebrados. In: Radostits O.M., Gay. C.C., Blood D.C. \& Hinchcliff K.W. (Eds). Clínica Veterinária. 9.ed. Rio de Janeiro: Guanabara Koogan, pp.1472-1547.

13 Riet-Correa F. \& Medeiros R.M.T. 2001. Intoxicações por plantas em ruminantes no Brasil e no Uruguai: importância econômica, controle e riscos para a saúde pública. Pesquisa Veterinária Brasileira. 21: 38-42.

14 Riet-Correa F. \& Méndez M.C. 1993. Introdução ao estudo das plantas tóxicas. In: Riet-Correa F, Méndez M.C. \& Schild A.L. (Eds). Intoxicações por plantas e micotoxicoses em animais domésticos. Pelotas: Hemisfério Sul, pp.1-20.

15 Riet-Correa F., Riet-Correa G. \& Schild A.L. 2002. Importância do exame clínico para o diagnóstico das enfermidades do sistema nervoso em ruminantes e eqǘ́deos. Pesquisa Veterinária Brasileira. 22: 161-168.

16 Rissi D.R., Rech R.R., Fighera R.A., Cagnini D.Q., Kommers G.D. \& Barros C.S.L. 2005. Intoxicação espontânea por Baccharis coridifolia em bovinos. Pesquisa Veterinária Brasileira. 25: 111-114.

17 Rodrigues R.L. \& Tokarnia C.H. 1995. Fatores que influenciam a toxidez de Baccharis coridifolia (Compositae): Um estudo experimental em coelhos. Pesquisa Veterinária Brasileira. 15: 51-59.

18 Schild A.L., Driemeier D., Barros C.S.L. \& Riet-Correa F. 2003. Plantas tóxicas para ruminantes e eqüinos no Rio Grande do Sul, Brasil. Boletim do Laboratório Regional de Diagnóstico. 23: 29-38.

19 Seitz A.L., Schwantes V.C., Schmitz M., Gimeno E.J., Barros S.S., Colodel E.M. \& Driemeier D. 2004. Intoxicação por Sida carpinifolia em ovinos. Pesquisa Veterinária Brasileira. 24 (Supl): 69. 
Pedroso P.M.O., Pescador C.A., Oliveira E.C., Sonne L., Bandarra P.M., Raymundo D.L. \& Driemeier D. 2007.Intoxicações naturais por plantas em ruminantes diagnosticadas no Setor de Patologia Veterinária... $\quad$ Acta Scientiae Veterinariae. 35:213-218.

20 Silveira A.L., Rocha A.O.B., Melo A.F.M., Santos E.J.V., Diniz M.F.F.M. \& Almeida Y.S. 2004. Plantas que ocorrem no Estado da Paraíba e suas potencialidades tóxicas. Pesquisa Veterinária Brasileira. 24 (Supl): 70.

21 Traverso S.D., Colodel E.M., Loretti A.P., Correia A.M. \& Driemeier D. 2003. Intoxicação natural por Trema micrantha em caprinos. Ciência Rural. 33: 133-136.

22 Traverso S.D., Corrêa A.M.R., Pescador C.A., Colodel E.M., Cruz C.E.F. \& Driemeier D. 2002. Intoxicação experimental por Trema micrantha (Ulmaceae) em caprinos. Pesquisa Veterinária Brasileira. 22: 141-147.

23 Traverso S.D., Corrêa A.M.R., Schmitz M., Colodel E.M. \& Driemeier D. 2004. Intoxicação experimental por Trema micrantha (Ulmaceae) em bovinos. Pesquisa Veterinária Brasileira. 24: 211-216.

24 Traverso S.D. \& Driemeier D. 2000. Experimental Trema micrantha (Ulmaceae) poisoning in rabbits. Veterinary Human Toxicology. 42: 301-302.

25 Tokarnia C.H., Döbereiner J. \& Peixoto P.V. 2000. Plantas Tóxicas do Brasil. Rio de Janeiro: Helianthus, 310p. 